\title{
Políticas públicas de adaptação à mudança climática na vida das mulheres nas áreas rurais do
}

\section{Nordeste}
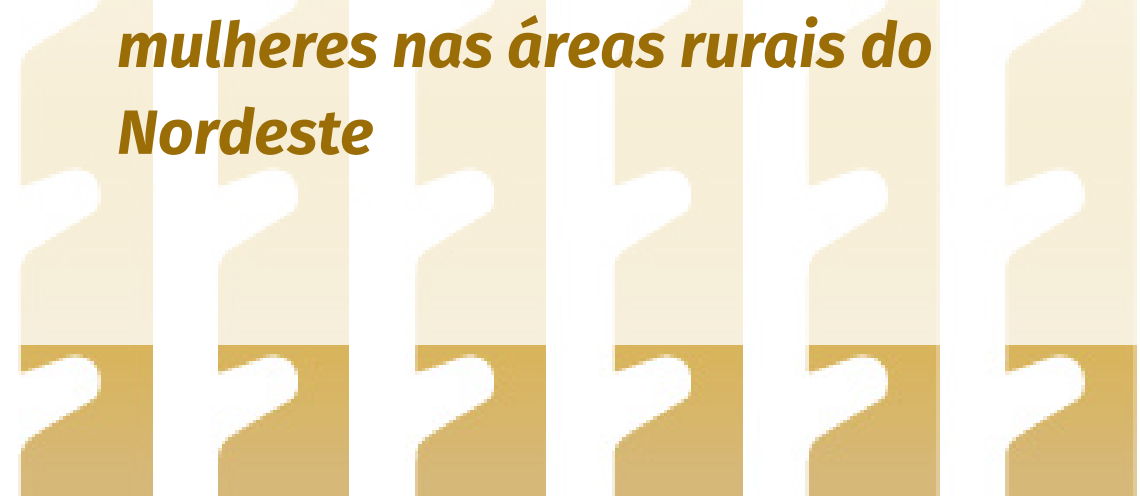

Raquel Barbosa Miranda*, Claudia Rangel** e Angelica Espinosa Miranda***

\section{INTRODUÇÃO}

O planeta Terra passou por muitas mudanças no clima desde o momento em que os seres humanos foram capazes de observá-las. Essas mudanças estão relacionadas à variabilidade natural (tais como alterações na radiação solar e dos movimentos orbitais da Terra) ou às atividades humanas diretas e indiretas que alteram os componentes da atmosfera global. Dados do Painel Intergovernamental de Mudanças Climáticas das Nações Unidas (IPCC), porém, indicam que a aceleração do aquecimento global que estamos vivendo hoje está diretamente relacionada à ação humana. As alterações climáticas são um desafio para o desenvolvimento sustentável e causam impactos não apenas no meio ambiente, mas também no desenvolvimento econômico e social (ONU, 1994). Segundo a Organização

"Aluna do Programa de pós-graduação - Master of Philosophy in Global development Theory and Practice Faculty of Psychology - University of Bergen (Noruega)

**Jornalista e Mestre em Educação - Universidade Federal do Espirito Santo (UFES)

***Professora Adjunta do Departamento de Medicina Social - Universidade Federal do Espirito Santo (UFES) 
das Nações Unidas (ONU), os efeitos da mudança climática varia não apenas de região para região mas, também, em função das diferenças sociais, ocupacionais, geracionais e entre mulheres e homens (ONU, 2009). Os países em desenvolvimento e as pessoas que vivem em situação de pobreza sofrerão impactos mais significativos dessa mudança (Hallegatte et al, 2016). No relatório do Banco Mundial Shock Waves: Managing the Impacts of Climate Change on Poverty, que avalia as relações entre a mudança climática e o empobrecimento, aponta que os seus desdobramentos (desastres naturais, perdas de safras e propagação de doenças) podem deixar mais de cem milhões de pessoas na pobreza, além de aumentar a incidência de doenças como malária, diarreia e nanismo, comprometendo seriamente a segurança da saúde da população. E esses efeitos serão mais sentidos nas regiões em desenvolvimento (Hallegatte et al, 2016). O Brasil é um dos países onde essas mudanças podem acarretar uma crise socioambiental de grandes dimensões; exemplo disso é a falta de chuvas que levou o país à pior crise hídrica dos últimos 84 anos, trazendo consequências econômicas e sociais negativas, principalmente na geração de energia elétrica e na agricultura (Ortiz, 2015). Os efeitos da mudança climática são particularmente evidentes nas comunidades empobrecidas, que sofrem mais diretamente com a redução da água potável e a com a diminuição da segurança alimentar provocadas por ela.

Os grandes desafios que as regiões politica e economicamente vulneráveis terão que enfrentar para minimizar os efeitos dessa mudança serão principalmente nos sectores econômicos, culturais e sociais, e colocarão em risco a segurança alimentar, a segurança humana e as condições básicas necessárias para a redução da pobreza (Oxfam, 2015; FAO, 2016). Seus impactos na América Latina são preocupantes, e afetam particularmente os setores rurais, como aponta o relatório do BIRD. Embora o Brasil seja uma importante potência agricola e industrial, a pobreza ainda é generalizada para a maior parte da população do país, e questões como desigualdade de renda e exclusão social permanecem sem solução (Buainain \& Neder, 2009). A re- 
gião Nordeste, a mais pobre e menos desenvolvida do país, é também a que mais sofre com os efeitos da mudança climática, pois grande parte da sua população depende da terra para garantir sua sobrevivência. Baixa renda, condições climáticas adversas e acesso limitado a serviços públicos levaram à migração de grande número de pessoas para áreas urbanas no sudeste do Brasil (Buainain \& Neder, 2009; IFAD, 2016).

Os problemas socioeconômicos e ambientais, porém, afetam de forma diferente os homens e as mulheres. As mulheres são particularmente vulneráveis em comparação com os homens, pois enfrentam desvantagens históricas, que incluem o acesso limitado à tomada de decisões e aos recursos econômicos necessários ao enfrentamento dos desafios provocados pela mudança climática (ONU, 2009). São, também, um dos grupos mais vulneráveis entre a população empobrecida das áreas rurais do Brasil. Composições familiares chefiadas por mulheres são muito comuns nas comunidades rurais, e isso ocorre por diversos fatores. Seja por causa da migração dos maridos para outras partes do país em busca de trabalho, ou pelo alto índice de famílias chefiadas por mães solteiras, cabe a essas mutheres a responsabilidade de administrar tanto a agricultura familiar quanto os domicílios (ONU, 2009; FIDA, 2016). Portanto, a análise de gênero deve ser aplicada a todas as ações relativas à mudança climática, e os pesquisadores de gênero devem ser consultados nos processos de enfrentamento de tais problemas em todos os niveis, para que as necessidades e prioridades especificas das mulheres e dos homens possam ser identificadas e abordadas (Mayesha, Bhatia, Mawby, 2015). No contexto das discussões de gênero e sua relação com a mudança climática, o objetivo deste artigo é descrever os impactos do Programa de Redução da Pobreza Rural (PCPR) no bem-estar das mulheres que vivem nas áreas rurais do Nordeste do Brasil. Este programa, financiado pelo Banco Internacional para Reconstrução e Desenvolvimento (BIRD) e tendo os estados como mutuários dos contratos, com o governo federal se restringindo a dar o aval às operações de financiamento, teve como 
propósito financiar a fundo perdido pequenos projetos de associações comunitárias organizadas, a fim de reduzir a pobreza em áreas rurais do país. Este projeto priorizou a integração entre os diversos setores com investimentos nas zonas rurais, por meio do planejamento participativo e monitoramento a nível local, municipal e estadual (IPEA, 2007).

Outras políticas de inclusão social e melhoria da vida das pessoas nas áreas rurais já haviam sido propostas anteriormente e outras surgiram na sequência do PCPR. As ações para a erradicação da fome e a diminuição da pobreza e das desigualdades sociais renderam ao Brasil amplo reconhecimento internacional. Essas ações são importantes e necessárias, principalmente quando se expande o olhar para as populações da zona rural (Mello, 2015). Os dados publicados no último Censo Demográfico 2010, apresentaram o maior peso relativo da pobreza na zona rural e uma diferença maior no acesso a bens e serviços quando comparados às áreas urbanas: a extrema pobreza atinge 25,5\% da população rural, enquanto no meio urbano ela cai para 5,4\%; o analfabetismo é de 20\% nas áreas rurais, contra 7,7\% na zona urbana; 52,9\% da população rural tem até quatro anos de escolaridade, enquanto na cidade esse número sobe para 25,9\% (Brasil, 2010). Diante desses dados, o eixo da inserção produtiva do plano Brasil Sem Miséria desenvolveu estratégias distintas para as áreas urbanas e rurais do país, com ações com características adequadas à variedade econômica e social da população rural. A população como um todo, e em particular as mulheres, foram beneficiados com as políticas voltadas para a melhoria das condições de vida durante a vigência do referido plano.

\section{OS DESAFIOS DA QUESTÃO DE GÊNERO NO ENFRENTA- MENTO À MUDANÇAS CLIMÁTICA}

A mudança climática é um desafio ao desenvolvimento sustentável, com impactos amplos não apenas sobre o meio ambiente, mas também sobre o desenvolvimento econômico e social (Babalola Oladapo \& Igbatayo Samuel, 2014). A redução da vulnerabilidade aos riscos climáticos é determinada principalmente pela capacidade de adaptação das 
pessoas (Kelly \& Adger, 2000). Algumas pessoas têm maior capacidade do que outras para administrar crises e um risco climático particular não afeta todas as pessoas dentro de uma comunidade com a mesma intensidade (Wilhitea, Sivakumarb e Pulwartyc, 2014). Há uma complexa inter-relação entre a capacidade de adaptação à mudança climática e a vulnerabilidade social e de gênero (ONU, 2011). A distribuição desigual de direitos, recursos, poder, regras e normas culturais restringe, para muitos, a capacidade de agir sobre os seus efeitos (CARE, 2015). Os impactos diferenciais dessa mudança sobre homens e mulheres são mais visiveis em lugares também afetados por conflitos violentos, instabilidade política e problemas econômicos (ONU, 2009; Mayesha et al, 2015).

Os conceitos de adaptação, mitigação, vulnerabilidade e resiliência estão inter-relacionados e têm uma aplicação importante para o enfrentamento da mudança climática. O IPCC define a adaptação climática como "um ajuste em sistemas naturais ou humanos em resposta a estímulos climáticos reais ou esperados ou seus efeitos, que prejudicam moderadamente ou exploram oportunidades benéficas". Enquanto a adaptação aborda os efeitos do fenômeno, a mitigação aborda as causas da mudança climática (IPCC, 2001; IPCC, 2014). A mitigação refere-se às ações de redução dos gases de efeito estufa (GEEs) liberados na atmosfera, para reduzir a quantidade de emissão e evitar as consequências desse acúmulo.(Smit, Burton, Klein e Street, 1999). A fim de orientar as políticas de adaptação e mitigação, muitos cenários possíveis dos efeitos dessas mudanças no clima global foram esboçados. No entanto, esses cenários apresentam limitações, em função da imprevisibilidade dos fenômenos climáticos naturais ou provocados (como os ciclos de retroalimentação e as vias de emissões) e das consequências sistêmicas não lineares do aumento das temperaturas globais (Machado Filho et al, 2016). Em relação à capacidade de adaptação das pessoas, é importante considerar o conceito de vulnerabilidade, definido pelo IPCC como a medida em que as mudanças climáticas podem danificar ou prejudicar um sistema, pois dela "de- 
pende não apenas da sensibilidade do sistema, mas também de sua capacidade de se adaptar às novas condições climáticas". Portanto, a definição de vulnerabilidade deve estar condicionada às estimativas dos potenciais efeitos da mudança climática e às respostas adaptativas (IPCC, 2001; IPCC, 2014). Assim, quanto menor for a vulnerabilidade e maior a resiliência, maior será a capacidade adaptativa da população. A resiliência pode transformar os problemas advindos da mudança climática em oportunidades de inovação e desenvolvimento de sistemas sócioecológicos, proporcionando a evolução de novos caminhos que melhorem a adaptação à essa mudança (Folke, 2006; Sharifi, 2016). Uma boa adaptação é igual a menor vulnerabilidade e maior resiliência; consequentemente, o aumento da capacidade adaptativa pode reduzir a vulnerabilidade e promover o desenvolvimento sustentável em muitas dimensões (Smit \& Wandel, 2006).

Embora o gênero seja um fator importante para a adaptação à mudança climática, seus aspectos raramente são abordados na elaboração das políticas de adaptação a elas. Há poucos dados, pesquisas ou estudos de caso esclarecendo e exemplificando as ligações entre a equidade de gênero e a mudança climática (Roehr, 2007). As mulheres são deixadas à margem do poder político e econômico e têm acesso limitado aos recursos financeiros e materiais, o que pode torná-las mais vulneráveis aos impactos da mudança climática (ONU, 2009). Os esforços para mitigar os efeitos dessa mudança e prevenir sua aceleração apresentam desafios sem precedentes, e um dos mais importantes deles está na desigualdade de gênero. Portanto, promover o empoderamento das mulheres e a igualdade de gênero é um dos pilares da construção de medidas para mitigar os efeitos da mudança climática (ONU, 2011). As mulheres que vivem em áreas rurais dos países em desenvolvimento, como o Brasil, são altamente dependentes dos recursos naturais locais para sua subsistência, devido à sua responsabilidade de economizar água, alimentos e energia para cozinhar e suprir outras necessidades da família (Mayesha et al, 2015). Algumas das consequências da mudança cli- 
mática, como a seca, as chuvas incertas e o desmatamento, tornam ainda mais dificil a tarefa de economizar esses recursos (Wilhitea et al, 2014). A mudança climática também coloca cargas adicionais sobre a saúde das mulheres e causa um efeito triplo sobre elas, pois, além de serem afetadas por suas vulnerabilidades fisicas especiais, também são afetadas por seus papéis de cuidado nas famílias e pelo trabalho adicional advindo do esgotamento dos recursos naturais. As alterações nas condições ambientais podem, portanto, levar a danos à saúde das mulheres (Roehr, 2007; ONU, 2009).

Embora todas as populações estejam vulneráveis aos impactos da mudança climática, o nível de vulnerabilidade difere significativamente nas regiões mais pobres, pois elas contam com menos recursos e menos influência. Esse é o caso do Nordeste brasileiro, onde a adaptação à mudança climática ainda é um grande desafio, pois interfere no bem-estar da população, causando-lhes desconforto e sofrimento no dia a dia. (Machado Filho, 2016). À medida que os riscos trazidos pela mudança climática e os distúrbios ambientais se tornam mais imediatos, também aumenta a necessidade de políticas para combater o problema (Nordhaus, 2007). As consequências da mudança climática devem crescer tanto em quantidade quanto em intensidade, e as políticas de adaptação voltadas para os pequenos agricultores devem levar em consideração os riscos da variabilidade climática. No Nordeste do Brasil, as mulheres das áreas rurais e pobres têm acesso limitado à educação, à mobilidade e aos recursos financeiros, o que evidencia e aumenta sua vulnerabilidade. Em muitos lugares, têm menos direitos civis, menos liberdade de ação, e suas vozes não são ouvidas quando se trata de formular políticas públicas ou mesmo de tomar decisões básicas em suas próprias casas. Além disso, é importante enfatizar que as áreas rurais do Nordeste dependem da agricultura de subsistência (Buainain \& Neder, 2009; Oxfam, 2015). São, portanto, ainda muito vulneráveis aos riscos decorrentes da seca e das mudanças no ciclo de chuvas causadas pela mudança climática (CARE, 2015). É certo que os homens também são 
afetados. Porém, aos homens é permitido, por exemplo, migrar em busca de aumentar a renda da família, enquanto que às mulheres resta adaptarem-se à nova realidade e cuidar das crianças, da pecuária e da agricultura. É ainda muito comum no semiárido brasileiro (região Nordeste) que os homens deixem suas terras em busca de melhores oportunidades nas grandes cidades, principalmente na região Sudeste, onde há mais oportunidades de emprego (Machado Filho et al, 2016). Enquanto isso, mulheres e crianças permanecem em casa. Isso ocorre porque, nessas regiões, culturalmente, o trabalho é organizado de acordo com o gênero; e dentro dessa organização tradicional do trabalho, cabe às mulheres o papel de cuidadoras do lar e da família. Com as secas causadas ou agravadas pelo aquecimento global, porém, o cuidado doméstico que as mulheres tradicionalmente exercem se amplia, pois elas têm que andar cada vez mais para buscar água e comida, o que tem um grande impacto sobre sua saúde e bem-estar.

A maneira como os projetos de adaptação à mudança climática voltados para a população rural ocorre no Nordeste do Brasil, porém, não garante a equidade de gênero. Reconhecer as diferentes necessidades e interesses, e exigir uma redistribuição de poder e recursos pode levar a uma mudança de fato transformadora. A igualdade de gênero está além da igualdade de oportunidades, pois aceita que mulheres e homens têm necessidades e interesses diferentes. Portanto, para promover a igualdade de resultados para toda a população do semiárido, é necessário que os projetos contemplem as diferenças entre homens e mulheres (Reeves \& Baden, 2000). Em outras palavras, é importante que as políticas e as medidas de adaptação à mudança climática se tornem sensíveis à questão do gênero.

\section{DISCUSSÕES CRÍTICAS SOBRE A SITUAÇÃO DAS MULHERES QUE VIVEM EM ÁREAS RURAIS NO NORDESTE DO BRASIL}

A literatura existente sobre mudança climática negligencia as implicações de paz e segurança deste desafio global e especificamente carece de uma discussão abrangente sobre 
os impactos da mudança climática nas diferenças de gênero (Roehr, 2007; Mayesha et al, 2016). É importante promover o financiamento de ações para a promoção da igualdade de gênero e o empoderamento das mulheres, especificamente no que se refere ao impacto da mudança climática sobre o bem estar delas. Também é importante integrar uma perspectiva de gênero ao planejamento, implementação, monitoramento, avaliação e relatórios das políticas ambientais nacionais, para além de fortalecer mecanismos fornecer recursos adequados para garantir a participação plena e igualitária das mulheres na tomada de decisões sobre questões ambientais, em todos os niveis (ONU, 2008). A Comissão da ONU sobre o Status das Mulheres considerou a questão da mudança climática em sua 46a sessão em 2002. As conclusões acordadas sobre a gestão ambiental e a mitigação de desastres naturais apontam para a necessidade de ações que incorporem a perspectiva de gênero às pesquisas sobre os impactos e causas das mudanças climáticas em andamento e encorajem a aplicação dos resultados destas pesquisas nas políticas e programas que visam minimizar esses impactos (ONU, 2002). A discussão seguiu a mesma direção na 52a sessão em 2008, quando a Comissão considerou a mudança climática como uma questão emergente e destacou o fato de que a mudança climática não é um fenômeno neutro de gênero, enfatizando seu impacto direto sobre as mulheres (ONU, 2008).

A região Nordeste do Brasil tem sido historicamente considerada uma das áreas mais pobres e subdesenvolvidas do país. Composta, principalmente, por pequenas fazendas de subsistência, além de algumas plantações de cana-de-açúcar, frutas e soja, tem uma população bastante dependente da agricultura como modo de vida, o que a torna ainda mais vulnerável aos impactos da mudança climática (Carless, 2014). As secas no Nordeste mataram milhares de pessoas e levaram a migrações forçadas para as cidades do Sudeste (Ortiz, 2015). Nos últimos vinte e três anos o Brasil vem adotando uma abordagem definida como Desenvolvimento Orientado pela Comunidade (CDD, traduzido da versão em inglês - Community-driven Development), para reduzir a 
pobreza rural na região. Essa é uma estratégia importante para reduzir a vulnerabilidade dessa população e, consequentemente, aumentar sua capacidade de adaptação aos efeitos da mudança climática. Encorajados por resultados rápidos, os governos estadual e federal continuaram a ampliar o programa de CDD, criando o "Programa de Combate a Pobreza Rural" (PCPR), que capacitou as comunidades locais para assumir o controle de seu próprio desenvolvimento e trabalhar em parceria com os governos estaduais e federal, o Banco Mundial, organizações não-governamentais e também com as universidades a fim de projetar e implementar investimentos que atendam à demanda real no campo em conformidade com o contexto local (Coiroloa \& Lammert, 2008).

Destacamos, aqui, as abordagens de gênero relativas aos efeitos do PCPR sobre as comunidades onde ele foi implementado. Essa avaliação foi baseada no projeto piloto "Redução da pobreza rural no Nordeste do Brasil: uma avaliação do desenvolvimento impulsionado pela comunidade", realizado no estado do Rio Grande do Norte, que foi projetado para fortalecer as ações focadas no gênero no âmbito do PCPR (Binswanger et al, 2009). Amazonas Barbosa, Costa e Romano (2011) analisaram um plano de ação sobre a questão do gênero com o objetivo de fazer uma avaliação exploratória indicativa dos efeitos do programa do Banco Mundial, que combinou investimentos em infraestrutura e abastecimento de água na comunidade com a produção orientada para o mercado. O projeto também criou um ambiente experimental para determinar quais seriam as estratégias mais apropriadas para incorporar questões de gênero e facilitar o empoderamento econômico das mulheres locais. O estudo estimou como os investimentos na comunidade diminuíram o tempo gasto pelas mulheres nas atividades domésticas e na busca por água, criando um contexto favorável para o empoderamento econômico dessas mulheres e ajudando a reduzir a pobreza nas comunidades onde o projeto foi aplicado. O projeto, que começou em 2009 e implementou 15 subprojetos em 12 comunidades, beneficiou 35 famílias na área selecionada. Os subproje- 
tos implementados incluíam sistemas de abastecimento de água potável e hortas produtivas (Amazonas et al, 2011). Em 2009, antes da implementação desse projeto, as atividades domésticas eram atribuídas principalmente às mulheres casadas. No entanto, os investimentos feitos nas comunidades estudadas reduziram essa carga para as mulheres, e isso parece ter ocorrido em decorrência de uma melhor distribuição das atividades domésticas entre mulheres casadas e jovens de ambos os sexos que eram solteiros. As duas principais hipóteses testadas durante o estudo constataram que a contribuição econômica das mulheres no orçamento familiar não era valorizada pelos padrões culturais tradicionalmente definidos por diferenças de gênero, e que, ao aumentar sua participação econômica, as mulheres também adquiriam mais consciência das desigualdades de gênero nos arranjos familiares e aumentavam seu poder de decisão.

Uma outra análise preliminar, que correlacionou a variação de renda e as dimensões culturais, sustentou a hipótese de que quanto maior a igualdade das contribuições econômicas no âmbito da família, mais equilibrado se torna o poder decisório familiar e menos patriarcal será a orientação sobre as questões de gênero. Verificou-se que a renda das mulheres cresceu, em comparação com os homens, em comunidades nas quais as perspectivas de gênero tornaram-se menos orientadas pelo patriarcalismo e nas quais a tomada de decisões domésticas tornou-se menos assimétrica em termos de gênero. Por outro lado, a renda das mulheres cresceu menos (em comparação com os homens) nas comunidades em que as perspectivas e atitudes relacionadas ao gênero e à tomada de decisões domésticas se mantiveram orientadas no modelo patriarcal e assimétricas em termos de gênero. Assim, parecia que um aumento na proporção da contribuição dos homens para os rendimentos familiares (em detrimento das contribuições das mulheres) levou as pessoas a atitudes mais patriarcais e desiguais em termos de gênero (Amazonas et al, 2011). O PCPR teve vários efeitos de curto prazo nas comunidades onde o projeto foi aplicado. O mais significativo foi o au- 
mento na renda das famílias que participaram dos projetos de produção agrícolas e artesanais, após capacitação adequada. Um ano após a implementação do projeto, o aumento da renda agricola proveniente das propriedades familiares participantes foi de $360 \%$, enquanto as famílias que não se beneficiaram do projeto aumentaram apenas $130 \%$ na renda agrícola. As evidências coletadas e analisadas durante o estudo sugeriram que o investimento no abastecimento de água aumentou o tempo livre das pessoas envolvidas na busca por água, particularmente entre as mulheres, permitindo que utilizassem seu tempo em outras atividades domésticas e agrícolas, que certamente aumentaram o bem-estar e a renda familiar (Binswanger et al, 2009). No entanto, não houve estudos para medir a quantidade de tempo economizado pelas mulheres, ou para identificar como as mulheres beneficiadas usaram o tempo que economizaram.

O PCPR trouxe muitos beneficios, que tiveram impacto positivo sobre a vida das mulheres das regiões rurais do Nordeste. No entanto, não trouxe certezas de que as mulheres puderam se beneficiar tão plenamente quanto os homens nos processos de CDD, pois as estruturas e normas patriarcais tornam suas vozes menos ouvidas do que as dos homens. A nível local, as desigualdades de gênero decorrentes dessas estruturas são um entrave para a participação de mulheres nos processos descentralizados e nas organizações comunitárias (Binswanger et al, 2009; Amazonas et al, 2011), e elas ainda têm menos acesso que os homens à tomada de decisão e aos recursos para o desenvolvimento (Negash, 2006). Esses fatos questionam a eficácia do projeto em relação à promoção do empoderamento e equidade entre mulheres e homens na região (Binswanger et al, 2009; Amazonas et al, 2011).

Se, por um lado, as abordagens de CDD resultaram em mais participação feminina na geração de renda familiar, por outro, essa inclusão nem sempre se traduziu em participação ativa e acesso igualitário aos beneficios para as mulheres. A mera disponibilidade de fundos, portanto, não 
é fator suficiente para garantir equidade de gênero. São necessárias, também, medidas adicionais específicas de empoderamento das mulheres mais pobres e vulneráveis do semiárido, para permitir que elas possam transformar suas necessidades em demandas efetivas e para que suas opiniões e prioridades sejam ouvidas nos processos coletivos de tomada de decisão (Baden, 2000; Goetz e Jenkins, 2005). As normas e padrões culturais da divisão social do trabatho e as relações de gênero que prevalecem na sociedade rural do Nordeste do Brasil são poderosos obstáculos para a inclusão e participação das mulheres (Cunningham, 2008; Binswanger et al, 2009). Na sociedade rural pobre do Nordeste brasileiro, as normas sociais tradicionais e o modelo patriarcal de organização familiar tornam o trabalho doméstico uma obrigação feminina, moldam uma distribuição assimétrica do poder na tomada de decisões intra-familiares, e privam as mulheres de oportunidades de participação em arenas civis e comunitárias de tomada de decisão (Oyediran \& Odusola, 2004; Amazonas et al, 2011). É importante observar que o PCPR não apenas diminuiu a pobreza em áreas rurais do Nordeste brasileiro, estimulando os pequenos agricultores a continuar trabalhando e cuidando de suas terras e contribuindo para a permanência das famílias em sua região original, mas também agiu de forma significativa para promover processos de adaptação à mudança climática na região.

Se compararmos a realidade da Região antes e após as intervenções realizadas no PCPR, com base nos dados do PCPR do Rio Grande do Norte, os resultados são animadores no que diz respeito ao crescimento do acesso à informação e aos indicadores de capital social. Porém, no que tange à governança, as instituições e as políticas públicas voltadas ao enfrentamento da mudança climática no Brasil, constatou-se que o espaço para questões relacionadas à agricultura é bastante limitado, especialmente para a agricultura familiar (Forbeloni, Nunes \& Costa, 2015; Machado Filho et al, 2016). Assim, para garantir que os interesses do setor sejam refletidos nas políticas e regulamentações, e na captação de recursos, é fundamental a implementação 
de políticas que visem a conscientização e a participação das partes interessadas nesse setor. A mentalidade precisa mudar, e os investimentos precisam ser canalizados para as pessoas das áreas rurais, que são as mais pobres e vulneráveis, para que se possa assim reduzir as desigualdades existentes (Buainain \& Neder, 2009; Machado Filho et al, 2016).

\section{CONCLUSÕES}

Mesmo sendo um grupo mais vulnerável à mudança climática, as mulheres podem representar a chave para resolver o problema (Mayesha et al, 2015), pois, muitas vezes, são elas as responsáveis por manter as famílias em tempos de crise. Cabe a elas lidar com a escassez e gerenciar os recursos para que eles durem. Os homens trazem o dinheiro, mas as mulheres são capazes de administrá-lo de uma maneira mais consciente. As mulheres são também mais capazes de fazer a renda familiar se multiplicar, pois tendem a investir não apenas em si mesmas, mas também na educação de seus filhos; sabem poupar os recursos e colocar comida na mesa a família. Isso ocorre não porque elas são naturalmente melhores nisso, mas porque têm séculos de aprendizado no gerenciamento familiar, que sempre foi atribuído, no modelo patriarcal, às mulheres. No geral, o PCPR foi benéfico para as mulheres das áreas rurais da região Nordeste do Brasil, no tocante à melhoria da renda e do bem-estar. No entanto, não é certo se o programa foi relevante se olhado através da lente de gênero, pois não há nenhum dado que comprove que as mulheres foram tão beneficiadas quanto os homens. Entretanto, os questionamentos das mulheres tem resultado em mudanças, mesmo que parciais, manifestadas no reconhecimento de direitos e na institucionalização das demandas como políticas públicas norteadas para a promoção da independência econômica. Ainda há um longo caminho a percorrer para que haja mudança na condição de invisibilidade da produção da mulher no meio rural, das diferenças na sua inclusão de renda familiar e na omissão por parte das políticas públicas, mas esforços contínuos tem sido feito nessa direção com o intuito de mudar o paradigma dessa situação. 


\section{REFERÊNCIAS:}

Amazonas F, Barbosa T, Costa A, Romano C. (2011) CDD Iniciativas para a Questões de Gênero no Nordeste do Brasil: Um Estudo de Caso Exploratório. Plano de Ação de Gênero, Agricultura e Desenvolvimento Rural. Escritório do Banco Mundial no Brasil. Unidade de Desenvolvimento Sustentável da América Latina e do Caribe. Banco Mundial.70p. Acessado em 21 setembro 17. http://documents.worldbank.org/curated/en/931281468223193690/pdf/718540PORTUGUEOniciativasOPortugues.pdf

Babalola Oladapo O, Igbatayo Samuel A. (2014) Climate Change and Poverty Nexus. International Scholarly and Scientific Research \& Innovation 8(9); 2864-2867.

Baden, S. (2000) Gender, Governance and The Feminization of Poverty, in Women and Political Participation: 21st Century Challenges. New York: UNDP.

Binswanger H, Amazonas F, T Barbosa, Costa A, N Menezes, Pazello E, Romano C. (2009) Rural Poverty Reduction in Northeast Brazil: An Evaluation of Community Driven Development. Vol. 2, Washington DC: World Bank. Acessado em 18 dezembro 2017. http://documents.worldbank. org/curated/en/166761468239374676/pdf/545100WPOVol2110Box349420B01PUBLIC1.pdf

Buainain, A. M. y Neder, H. D. (2009) Crisis y pobreza rural en América Latina: el caso de Brasil". Documento de Trabajo N³8. Programa Dinámicas Territoriales Rurales. Rimisp, Santiago, Chile. Acessado em 5 outubro 2016. www.rimisp.org/dtr/documentos.

Brasil (2010). Censo demográfico Nacional 2010. Instituto Brasileiro de Geografia e Estatística. Acessado em 24 abril 2018. https://censo2010.ibge.gov.br

Care (2015) Adaptation, gender and women's empowerment. CARE International Climate Change Brief. 14 dezembro 2017. http://www.care.org/sites/default/files/documents/CC-2010CARE_Gender_Brief.pdf

Carless W. (2014) The biggest disaster you've probably never heard of. Global Post, Feb 14, 2014. Acessado em 26 outubro 2016. http://www.pri.org/stories/2014-02-14/biggest-disaster-you-ve-probably-never-heard.

Coirolo, L, Lammert J. (2009) Rural poverty reduction in Northeast Brazil: Achieving results through community driven development. Washington, DC: World Bank.

Cunningham M. (2008) Influences of gender ideology and housework allocation on women's employment over the life course. Soc Sci Res; 37 (1): 254-267.

FAO (2016) Organização das Nações Unidas para a alimentação e agricultura. América Latina e Caribe no caminho certo para erradicação da fome e da pobreza. Acessado em 25 setembro 2016. http://www.fao.org/news/story/pt/item/385290/icode/

Folke C. (2006) Resilience: The emergence of a perspective for social-ecological systems analyses. Global Environmental Change. 16: 253-267.

Goetz AM, Jenkins R. (2005) Reinventing Accountability: Making Democracy Work for Human Development. Baskingstoke: Palgrave Macmillan. Acessado em 21 outubro 2017. http:/ /www. palgraveconnect.com/pc/doifinder/view/10.1057/9780230500143.

Hallegatte S, Bangalore M, Bonzanigo L et alii (2016) Shock Waves: Managing the Impacts of Climate Change on Poverty. Climate Change and Development Series. Washington, DC: Wor- 
Id Bank. doi:10.1596/978-1-4648-0673-5. License: Creative Commons Attribution CC BY 3.0 IGO Acessado em 12 dezembro de 2017.

https://openknowledge.worldbank.org/handle/10986/22787

IFAD. (2016) International Fund for Agricultural Development. Investing in rural people in Brazil. Acessado em 25 setembro 2016. https://www.ifad.org/documents/10180/f5eb1cd0-a412-45bc-9218-1dc9fd7876b5

Forbeloni J.V., Nunes E.M \& Costa J.B.A (2015). Politicas publicas de combate à pobreza rural na microrregião de Angicos, RN: uma proposta de Segurança Protetora na perspectiva do Desenvolvimento como Liberdade. VII Jornada Internacional de Políticas Públicas. Acesso em 17/04/2018. http://www.joinpp.ufma.br/jornadas/joinpp2015/pdfs/eixo4/politicas-publicas-de-combate-a-pobreza-rural-na-microrregiao-de-angicos.pdf

IPEA (2007). Programas governamentais de combate à pobreza rural no Nordeste. Acessado em 18 abril 2018.

http://www.ipea.gov.br/agencia/images/stories/PDFs/livros/07Cap01_rogramasGover.pdf

IPCC. (2014) The Intergovernmental Panel on Climate Change 2014. Impacts, Adaptation, and Vulnerability Summaries, Frequently Asked Questions, and Cross-Chapter Boxes. Acessado em 18 setembro 2016. http://www.ipcc.ch/report/ar5/wg2/

IPCC. (2001) The International Panel on Climate Change 2001. Impacts, Adaptation and Vulnerability. Contributions of the Working Group III to the Third Assessment Report of the Intergovern- mental Panel on Climate Change. Cambridge.

Kelly PM, Adger WN. (2000) Theory and practice in assessing vulnerability to climate change and facilitating adaptation. Climatic Change 47: 325-352.

Machado Filho H; Moraes C; Bennati P; Rodrigues RA et alii (2016) Climate change and impacts on family farming in the North and Northeast of Brazil. Acessado em 3 outubro 2016.http:// www.ipcndp.org/pub/eng/WP141_Climate_change_and_impacts_on_family_farming.pdf.

Mayesha AM, Bhatia R, Mawby B. (2015) Women and Climate Change: Impact and Agency in Human Rights, Security, and Economic Development. Acessado em 22 setembro 2016.

https://giwps.georgetown.edu/sites/giwps/files/Women\%20and\%20Climate\%20Change.pdf

Mello, J. (2015) A inclusão produtiva rural no Brasil Sem Miséria: o desafio da superação da pobreza no campo. Brasília, DF: MDS. (p. 17-31) (Cadernos de Estudos Desenvolvimento Social em Debate no 23). Acessado em 20 abril 2018. http://aplicacoes.mds.gov.br/sagirmps/ferramentas/docs/Caderno\%2023_\%20FINAL_910.pdf.

Negash A. (2006) Economic Empowerment of Women. Santa Clara University. Acesso em 12 dezembro 2017. https://www.scu.edu/ethics/focus-areas/more/resources/economic-empowerment-of-women/

Nordhaus WD. (2007) A Review of the Stern Review on the Economics of Climate Change. Journal of Economic Literature, Vol. XLV, pp. 686-702.

ONU. (1994) The United Nations Framework Convention on Climate Change. 21 March 1994. Accessado em 28 setembro 2017. http://newsroom.unfccc.int

ONU. (2002) 46th Session of the Commission on the Status of Women (2002) "Agreed Conclu- 
sions". Report on the forty-sixth session of Commission on the Status of Women. Official Records, 2002 supplement No. 7 (E/2002/27-E/CN.6/2002/13). p12. Economic and Social Council, United Nations.

ONU. (2008) 52nd session of the Commission on the Status of Women (2008) "Gender perspectives on climate change," Issues paper for interactive expert panel on Emerging issues, trends and new approaches to issues affecting the situation of women or equality between women and men.

ONU. (2009) Women, Gender Equality and Climate Change. Accessado em 28 setembro 2017. http://www.un.org/womenwatch/feature/climate_change/

ONU. (2011) Overview of linkages between gender and climate change. Acessado em 5 outubro 2016. http://www.undp.org/content/dam/undp/library/gender/Gender\%20and\%20Environment/PB1_Africa_Overview-Gender-Climate-Change.pdf.

Ortiz F. (2015) Brazil faces water rationing amid worst drought in 84 years. Accessado em 28 setembro 2016. http://www.climatechangenews.com/2015/02/16/brazil-faces-water-rationing-amid-worst-drought-in-84-years.

Oxfam. (2015) How rural women are adapting to climate change in Latin America and the Caribbean. Accessado em 25 setembro 2017. https://www.oxfam.org/en/peru-brazil-nicaragua-cuba-mexico-bolivia-el-salvador-dominican-republic/how-rural-women-are.

Oyediran KA, Odusola AF. (2004) Poverty and the Dynamics of Women's Participation in Household Decision-Making in Nigeria. African Population Studies Suppl. A to vol 19:115-139.

Reeves H, Baden S. (2000) Gender and Development: Concepts and Definitions. Prepared for the Department for International Development (DFID) for its gender mainstreaming intranet resource. BRIDGE, 38p:pages 10-11.

Roehr U. (2007) Gender, climate change and adaptation. Introduction to the gender dimensions. Accessado em 5 de outubro 2017. http://www.unep.org/roa/amcen/Projects_Programme/climate_change/PreCop15/Proceedings/Gender-and-climate-change/Roehr_Gender_climate.pdf.

Sharifi A. (2016). A critical review of selected tools for assessing community resilience. Ecological Indicators. 69: 629-647. doi:10.1016/j.ecolind.2016.05.023.

Smit B, Wandel J. (2006) Adaptation, adaptive capacity and vulnerability." Global environmental change 16.3: 282-292.

Smit B; I. Burton; R.J.T. Klein; R. Street. (1999) The Science of Adaptation: A framework for Assessment". Mitigation and Adaptation Strategies for Global Change. 1999, 4: 199-213. doi:10.1023/A:1009652531101.

Wilhitea DA, Sivakumarb MVK, Pulwartyc R. (2014). Managing drought risk in a changing climate: The role of national drought policy. Weather and Climate Extremes. Volume 3, P4-13. http://dx.doi.org/10.1016/j.wace.2014.01.002. 